\title{
La Tradizione Giuridica Indigena ${ }^{1}$
}

\author{
The Indigenous Legal Tradition
}

\section{Carlos Humberto Durand Alcántara}

Universidad Autónoma Metropolitana, Azcapotzalco, México

\begin{abstract}
Resumo: Il tema principale di questo lavoro si riassume nel tentativo, in primo luogo, di spiegare la natura dei rapporti giuridici che si sviluppano nelle realtà indigene, e se è possibile identificarli come un "sistema legale". In secondo luogo, si cercheranno di individuare gruppi sociali nei quali vige un modello legale pluralista. La struttura e i metodi dei sistemi di diritto indigeni formano un tutto indissolubile con la "visione del mondo" di questi stessi popoli, con la loro cultura, arrivando così a rifletterne le specifiche identità.
\end{abstract}

Parole chiave: Indigena. Giuridica. Tradizione.

\begin{abstract}
The main theme of this work is summarized in the attempt, first, to explain the nature of the legal relationships that develop in the Indigenous peoples reality, and if you can identify as a "legal system." Secondly, we will try to identify the social groups that have imposed a legal pluralist model. The structure and methods of indigenous legal systems constitute an indivisible whole with the "world view" of these same people, and your culture, thus establishing their specific identities.
\end{abstract}

Keywords: Indigenous. Legal. Tradition.

\section{Introduzione}

Per la prima volta, nelle sue Risoluzioni del 1994 la Commissione per i Diritti Umani della Organizzazione delle Nazioni Unite ${ }^{2}$ riconobbe che le pratiche giuridiche delle popolazioni indigene del mondo costituis-

\footnotetext{
1 Recebido em: 24/02/2014

Revisado em: 15/04/2014

Aprovado em: 16/05/2014

2 Ci riferiamo alla $46^{\mathrm{a}}$ sessione della Sottocommissione per la Lotta alle Misure Discriminatorie e la Tutela delle Minoranze, e conforme al Rapporto del gruppo di lavoro sulle popolazioni indigene E/CN.4/Sub.2/1994/2/Add.1, del 20 aprile del 1994.

Fin dalle sue origini, la Commissione per i Diritti Umani della ONU, che fu presieduta dal distinto giurista Abjorn Eide, orientò l'adeguamento dei diritti indigeni come tradizione, Cfr. Bozza della Dichiarazione sui Diritti Indigeni del 1985 e le seguenti modifiche del documento fino al 1993.
} 
cono sistemi di diritto. In merito, l'articolo quarto della futura Dichiarazione Universale sui Diritti dei Popoli Indigeni stabilisce: "I popoli indigeni hanno diritto a mantenere e rafforzare le loro particolari istituzioni politiche, economiche, sociali e culturali, così come i loro sistemi legali [...]".

Questo nuovo adeguamento dei diritti indigeni osserva, contrario sensu ai fini della globalizzazione, che lo Stato post-moderno capitalista riconoscerà l'esistenza di diversi sistemi di diritto che confluiscono congiuntamente in quello egemonico, ciò che alcuni autori hanno definito come "pluralismo giuridico"; tuttavia ci si dovrà chiedere in che misura, dentro uno stesso territorio, la sovranità dello Stato permetterà l'applicazione, efficace, di diversi sistemi di diritto ${ }^{3}$, in questo caso quelli che corrispondono alle popolazioni indigene. È evidente che al fondo di questo dibattito sottende un problema di potere.

In questo modo, rappresenta una sfida per le scienze sociali, per $\mathrm{i}$ popoli indigeni e i loro intellettuali organici stabilire gli argomenti che spieghino a sufficienza questo nuovo adeguamento degli "altri sistemi di diritto" e la loro fattibilità nel mondo post-moderno; aspetto, questo, che in qualche modo contribuirà al rafforzamento del quadro concettuale della futura Dichiarazione sui Diritti Indigeni da parte dell'Assemblea generale dell'ONU.

\section{Il Soggetto Sociale Indigenae L'Applicazione del suo Sistema di Dirito}

Chi sono i soggetti sociali indigeni, comunità, popolazioni e nazioni indigene alle quali corrisponde questo nuovo adeguamento sistematico dei loro diritti?

Tradizionalmente la questione indio-indigeno è stata spiegata in relazione agli abitanti originari dell'America e ai loro discendenti; tutta-

\footnotetext{
3 Per Carbonier (1978, p. 10), la coesistenza di un diritto statale con altri diritti può essere solamente un'illusione. Questa coesistenza corrisponde ad un pluralismo giuridico da sopravvivenza.
} 
via questo concetto - chiamiamolo descrittivo (ciò che è indio) - ha una connotazione molto più ampia che propriamente americana, cosicché con un concetto universale la ONU segnala:

Comunità, popoli e nazioni indigene sono quelle che $[. .$.$] conside-$ rano se stessi differenti dagli altri settori della popolazione [...]. Per il momento essi non fanno parte dei settori dominanti della società e sono fermamente decisi a mantenere i loro territori ancestrali e la loro identità etnica. (HUMAN RIGHTS, 1991, p. 6)

Secondo questa concezione, i "popoli indigeni" si trovano nei cinque continenti ${ }^{4}$ e in più di 75 Paesi. Così gli "ainu" e i "barakumin" del Giappone, i "dai", "miao", “yi”, "yugur", "kiriguiz" -tra gli altridella Cina (DAI CHA FOU, 1991, p. 79-92); gli “adivasi” dell'India, i "papúas", i “dayak", i "penán”, e gli "aetas" nell’Asia Sudorientale, i pigmei "batawa" e "twa" del Ruanda e, incluso, gli aborigeni australiani o gli "inuit" di Alaska, Canada e Groenlandia sono indigeni. La vecchia concezione di riconoscere come indio tutto ciò che è americano è oggi senza fondamento. I "quasi mille" popoli d'America non rappresentano che un quarto dell'intera popolazione indigena del mondo. Così, il 90\% della diversità culturale nel mondo si deve alle popolazioni indigene. Circa 250 milioni di persone, vale a dire il $4 \%$ della popolazione mondiale, appartengono a uno dei gruppi indigeni sopravvissuti. ${ }^{5}$

4 Come indicato, il termine indigeno s'incorporò nell'anno 1762 nel Dizionario dell'Accademia Francese, che al rispetto segnala: "Indigene. Subst. Il se dit des naturels d'un pays". (ALCIDES REISSNER, 1980)

5 Tuttavia, secondo uno studio del Massachusetts Institute of Technology (MIT) nella prossima generazione scomparirà la metà dei 6000 idiomi che attualmente si parlano nel pianeta; 3000 lingue sono destinate a morire perché nessun bambino le parla più. Solo in Papua Nuova Guinea si parlano 800 differenti idiomi, in Indonesia circa 240, in tutta l'Africa più di 800 . Secondo il MIT solamente 300 idiomi di questo tesoro linguistico avranno un certo futuro. Questo è un disastro per l'umanità perché per ogni idioma che scompare svanisce una cultura locale e perdiamo un mondo. Nozioni filosofiche e religiose insostituibili e generazioni di conoscenza sulla vita in terra che si perdono. (HEIKE; VEREYEN, 1993, p. 4) 


\section{Verdo un Adeguamento Epistemologico del Diritto Indigeno}

D'accordo a Werner Krawietz (1979, p. 113-152, 181) consideriamo che nella teoria generale del Diritto esiste una nuova visione metodologica, epistemologica e paradigmatica del Diritto, la quale sorge come una critica al dogmatismo giuridico, il cui fondamento è quello di comprendere $\mathrm{i}$ fenomeni giuridici a partire dalla prassi giuridica.

La discussione centrale di questo saggio si colloca nel tentativo di spiegare il significato peculiare che acquisiscono i rapporti giuridici che si sviluppano nella realtà indigena, e se a partire da ciò sia possibile riconoscere il diritto consuetudinario indigeno come un "sistema legale". ${ }^{6}$ Un secondo fondamento radica nell'individuare l'esistenza di formazioni sociali alle quali sottenda un modello pluralista del diritto.

La costruzione epistemologica dei sistemi di diritto indigeni forma parte di un insieme di categorie più ampie, come, tra le altre, la cosmovisione (la "visione del mondo", n.d.t.) ${ }^{7}$ e la cultura dei popoli indigeni, che a loro volta ci permettono di riconoscere l'identità di gruppo che soggiace a cadauno di essi.

In questo modo, all'identificare un sistema di diritto indigeno è indispensabile adeguarlo all'ambiente socioculturale nel quale si conosce e si applica.

Le ricerche circa i sistemi normativo-giuridici indigeni, vale a dire la spiegazione integrale dell'insieme di regole, principi, norme azioni, procedimenti e organi esecutori del diritto indigeno, non hanno raggiunto la piena maturità.

\footnotetext{
6 Per dare fondamento al concetto di sistema legale utilizziamo la scuola contemporanea del diritto francese, la quale definisce che: "Ogni diritto costituisce di fatto un sistema, impiega certo vocabolario, corrispondente a determinati concetti; raggruppa le regole in determinate tecniche per formulare le norme e determinati metodi per interpretarle; è legato a una determinata concezione dell'ordine sociale, che definisce il modo di ampliare e la funzione stessa del diritto". Sebbene nei limiti del quadro struttural-funzionalista, questa categorizzazione ci permette di avvicinarci alla delimitazione del nostro oggetto di studio. (DAVID; JAUFFRET-SPINOSI,1988, p. 20)

7 Voglio dire al Maestro Elvis de Biasi, caro amico, il nostro profondi ringraziamenti per la perseveranza.
} 
Tra gli sforzi più significativi si trovano: la scuola olandese del diritto consuetudinario, che propose la creazione della Commission of Falk-Law and Legal Pluralism. Tra i lavori di questa scuola si evidenziano: John Griffits, et. al., Antropology of law in the Netherlands, Essays on Legal Pluralism (1986). Nella scuola anglofona i lavori di Laura Nader, The Antropological Study of Law (1965) e On Study the Ethnography of Law and its consecuences. In Asia, Asia Indigenous-Law, di M. Chiba (1986); sul diritto consuetudinario africano, Ideas and Procedures in African Costumare Law, di Max Gluckman; in Francia, i lavori in quest'area si sviluppano principalmente nei Laboratoires de Antropologie Juridique, diretti da Michel Alliot ed Etienne Leroy, e nel Centre Droit et Cultures (1977), diretti da Raymond Verdier. In Australia, i lavori di Irene O'Connell. In America Latina i lavori di Rodolfo Stavenhagen; in Guatemala, le ricerche del Dott. Rolando López Godínez e infine, in Messico, i lavori dell'Istituto di Ricerche Giuridiche della UNAM, guidati dal Dott. Jorge Alberto González G., e dell'Università di Chapingo, attraverso i lavori del Dottorato di Sociologia Rurale, corrispondenti al progetto si popolazioni indigene, coordinato dal Dott. Carlos H. Durand.

\subsection{Alcuni Argomenti Metodologici per la Comprensione dei Sistema di Dirito Indigeno}

A nostro parere, il trattatista del fenomeno in questione conta su tre fondamenti metodologici:

a) Quello del marxismo, che si fonda sul formen (modo di produzione) e le sue rispettive relazioni di produzione, le quali interagiscono con una sovra-struttura storicamente determinata.

b) Quello struttural-funzionalista, che interpreta la natura dei fenomeni culturali come funzioni organizzate in istituti e volte generalmente alla risoluzione di una determinata necessità. In quest'ottica, il sistema di diritto indigeno aspira a mantenere "l'ordine sociale e l'unità del gruppo".

c) Quello simbolico, che si basa nell'idea delle pratiche giuridiche come simboli che generalmente hanno un significato che ci conduce in zone al di là delle pratiche stesse. Quindi, gli scontri 
sono simboli che esprimono la gerarchia sociale e la "visione del mondo" di qualche etnia in particolare.

Il nostro oggetto di studio si colloca ai margini della Teoria Generale del Diritto e dello Stato, così come nel campo dell'Antropologia (e al suo interno, dell'Etnologia) e, ovviamente, della Sociologia Giuridica. Avvertiamo quindi un approccio interdisciplinare dello studio del sistema di diritto indigeno.

Con tutte le difficoltà che ciò rappresenta, dato l'oggetto di studio proposto, cercheremo di costruire una visione che, ambiziosamente, definiremo "olistica" e nella quale è rilevante la categoria di "cultura", circostanza che ci pone nel campo dell' antropologia giuridica e della sociologia giuridica.

Compito complesso risulta essere quello di consolidare formulazioni compiute rispetto al fenomeno studiato; tuttavia, una volta affermatane la certezza, rispetto all'esistenza del diritto indigeno, si spiegheranno alcuni degli aspetti che risultano trascendenti per il suo adeguamento.

Lo studio delle normatività indigene non può essere elaborato secondo modelli positivisti o "scientificisti", per i quali la norma giuridica si spiega solamente come un potere egemone dello Stato o come una transizione evolutiva dall'uso alla norma.

I processi sociali concreti che si danno nelle popolazioni indigene permettono di constatare che i sistemi giuridici esistono come fenomeno specifico, costituendo un aspetto rilevante nella regolazione della realtà sociale dei citati popoli, e i cui fondamenti radicano nella loro cultura, differente dai modelli occidentali.

Nel definire la normatività giuridica indigena come un sistema per se, è pertinente provare la ricerca di quelle regolarità che probabilmente lo spieghino, stabilendo alcune delle categorie con le quali sarebbe possibile avvicinarsi al suo significato. Al rispetto abbiamo trovato cinque categorie:

a) le società semplici;

b) i rapporti di proprietà; 
c) le autorità come organi applicatori del diritto;

d) il linguaggio;

e) la parentela.

Concetti che spiegheremo in modo analitico, cercando di non perdere la visione d'insieme del fenomeno in oggetto.

\subsubsection{Società Semplici e Popoli Indigeni}

La società semplice è lo spazio territoriale nel quale acquista efficacia e validità (sebbene in alcuni casi relativa) l'applicazione della normatività indigena. ${ }^{8}$

La società semplice non suppone una comunità auto-contenuta e autarchica, al di fuori della crescita capitalista, ma piuttosto l'esistenza di popolazioni indigene che, in diversa misura, interagiscono con la società complessa e nei cui territori esistono diritti consuetudinari (MARX, 1984) che sono applicati e riconosciuti solamente all'interno dei loro confini.

Originalmente queste società semplici risalgono alle forme "primitive" che storicamente aprirono il cammino allo sviluppo della società umana. Lewis Morgan, nel quadro dell'antropologia evoluzionista, spiegò gli sviluppi di queste società -aspetto ripreso da Carl Marx (1984), non solo nelle sue opere classiche che allusero al modello di produzione comunista primitivo ma piuttosto nella critica dello schema delle formen (Grundrisse) nel quale lo stesso Marx riconobbe problemi critici dell'evoluzione storica- individuando diverse comunità primitive che si sono trasformate in modo eterogeneo verso distinte forme di Stato e società in classi, approfondendo i suoi studi in quello che lui stesso definì come "modello di produzione asiatico".

Nei Grundrisse Carl Marx (1984, p. 12) riferisce: “L'uomo si isola solo attrraversoil proceso storico. Originariamente egli si presenta come esseresociale, tribale, animale gregario. È una comunità di sangue, di lin-

\footnotetext{
8 L'uso del termine "costume giuridico indigeno" non rappresenta una semplice questione semantica bensì va oltre, nel senso di riconoscere il profilo storico dei sistemi di diritto indigeno, i quali non possono essere compresi al margine della loro consuetudinarietà, che si è venuta reinterpretando nel tempo.
} 
gua, di costumi e deve esistere previamente perché l'individuo si appropri delle condizioni oggettive di vita". In America Latina le società semplici sono alla base delle etnie.

L'etnia costituisce un'unità territoriale con spazi interni delimitati e gerarchizzati: quartieri o rioni, paraggi, siti. L'etnia come tale si regge su una serie di principi di autogestione e una relativa uguaglianza, il che comporta una forma molto particolare di praticare la democrazia. L'etnia tende a regolare al suo interno tutti gli aspetti della vita sociale, economica, culturale e religiosa; compresa la terra, la cui distribuzione e uso, in questo sistema, sono sanzionati dalla comunità, dal momento che essa da e toglie nel rispetto di certe leggi interne.

\subsubsection{Rapporti di Proprietà Indigeni}

I rapporti di proprietà intesi come l'espressione giuridica dei rapporti di produzione che, storicamente, le etnie hanno sviluppato per soddisfare le loro necessità e interagire con gli altri settori e classi sociali della società, non possono, dal punto di vista metodologico, essere separati, nello studio dei sistemi di diritto indigeni, dalla loro matrice socioeconomica, viste le particolarità che integrano il suddetto diritto.

La normatività giuridica delle popolazioni indigene si collega alle forme di appropriazione sociale dell'ambiente, così come alla loro realtà sociale e cosmovisione, ed esistono tante interpretazioni culturali d'accordo a cadauna delle etnie esistenti al mondo; proposto in questi termini, lo studio dei sistemi di diritto indigeni acquisisce validità nell'assunto epistemologico della totalità, all'osservare la pertinenza di fenomeni che s'intrecciano e spiegano in forma globale.

I sistemi giuridici consuetudinari dei popoli indigeni si basano in un tacito riconoscimento (interno) dei rapporti di proprietà , i quali si manifestano in due modi:

a) la proprietà comunale dello spazio físico (la terra), regolata attraverso uno "statuto comunale" (di tradizione orale), che può essere sfruttato da tutta la comunità (acque, boschi, fauna ecc.); 
b) la proprietà familiare, che si attribuisce nella parentela e che allude ai mezzi di produzione, agli strumenti di lavoro, alberi piantati, animali domestici eccetera.

La comunità crea e ricrea l'insieme di tradizioni giuridiche che permeano i rapporti di proprietà.

Quindi, anche se riconosciamo l'ipotesi secondo cui i popoli indigeni sono possessori di "culture giuridiche proprie" che regolano, secondo la loro identità, i rapporti sociali di produzione e di proprietà, è importante precisare che i sistemi giuridici indigeni non costituiscono un ordinamento isolato, "puro", bensì compenetrato da altre e diverse relazioni storiche (economiche, giuridiche, politiche, religiose, linguistiche) come dal sistema giuridico del blocco dominante.

Piuttosto, l'asserzione di riconoscere l'esistenza dei sistemi giuridici consuetudinari si colloca ai margini del concetto di autonomia relativa, che spiegherebbe la sopravvivenza dei sistemi di diritto indigeni nell'interazione delle due società e nonostante l'egemonia di una di esse.

I rapporti comunitari di proprietà, organizzazione sociale, parentela, e le loro forme di riproduzione sociale, sono la matrice di esperienze in cui si riproducono e interagiscono i più volte citati sistemi di diritto indigeni.

Tuttavia, nel caso particolare delle popolazioni indigene, la matrice socioeconomica dei rapporti di proprietà dev'essere studiata con somma attenzione posto che la terra, per questi popoli, non è intesa solamente come un semplice mezzo di produzione ma come fondamento e base della loro stessa esistenza spirituale.

D'altra parte, i rapporti di proprietà dei popoli indigeni si basano nell'identità culturale del gruppo. È solamente come membro di una comunità che un indigeno può incorporarsi ai rapporti di proprietà del nucleo sociale.

L'identità culturale dell'etnia permette di riconoscere altre categorie significative, quali la parentela, il linguaggio, la religione eccetera, elementi coadiuvanti nella comprensione del diritto indigeno. Riferendosi 
all'integrazione di queste categorie e alla tradizione indigena, Mario Rizo (1991, p. 10) menziona:

Il diritto consuetudinario non per le origini diverse che manifestino le sue norme deve considerarsi come un'amalgama informe e incoerente; al contrario, l'efficacia che in questo diritto hanno le citate norme è espressione di un processo di organizzazione analogica propria che, sul piano culturale, ideologico o semiotico, realizza la comunità in base a quelli che essa stessa definisca come suoi interessi basici, materiali e immateriali.

\subsubsection{Il Potere e l'autorità nelle Popolazioni Indigene}

È logico supporre che, se il diritto indigeno (e qualsiasi tipo di diritto) esiste come un sistema efficace, esisterà congruentemente l'istanza che regolerà e amministrerà la suddetta normatività.

Collocandosi nello studio delle società più remote, Clastres (1984, p. 207) precisa:

[...] gli studi di campo sulla società primitiva, la società senza Stato, mostrano che, al contrario, la prima divisione non è quella tra gruppi sociali opposti, tra ricchi e poveri, sfruttatori e sfruttati, ma quella che serve da base a tutte le altre divisioni, quella che esiste tra quelli che comandano e quelli che obbediscono, perché la cosa fondamentale è la divisione della società tra quelli che esercitano il potere e quelli che sono sottomessi ad esso.

Sosteniamo l'ipotesi che è falso supporre l'idea che tutta la tradizione giuridica si sia evoluta fino a convertirsi in norma giuridica, come sostiene in qualche modo la concezione positivista del diritto; piuttosto è esistita una rottura storica che ha determinato il cambio nella natura giuridica di quel diritto, da una tradizione normativa che si basava sulla reciprocità e sull'oralità si passò a una normatività delle egemonie, mentre il diritto indigeno è sopravvissuto "marginalmente". In altri termini, si trasformò anche il senso di autorità superiore. 
L'esercizio dell'autorità che regola ed applica il diritto indigeno si colloca in due sfere: una che lo delimita ai confini dell'etnia e quella in cui interagisce con l'autorità egemone (quella del blocco dominante).

\subsubsection{Esiste una Legittimazione Consensuale dell'autorità Indigena?}

L'applicazione del diritto indigeno, e la sua efficacia, si origina nel presupposto di un'accettazione dell'autorità che si basa in un insieme di scambi reciproci. A differenza del potere di classe, il senso dell'autorità delle comunità e di alcune etnie nasce da un'accettazione reciproca per la quale i loro membri riconoscono che uno qualsiasi di loro potrà eventualmente formar parte della struttura che applicherà il diritto. Forse in buona misura questo senso di reciprocità è uno degli elementi che ha permesso di mantenere, con maggior o minor forza, le autorità indigene di alcune etnie nel mondo. Tuttavia, lo studio del potere in una determinata regione richiede di adeguare l'analisi dell'equilibrio precario tra i sistemi politici indigeni e la penetrazione del capitale, il cui epicentro radica nel non-riconoscimento del potere di dette autorità; vorremmo insistere allora sul problema dell'egemonia e sul fatto che, per alcuni studiosi, il rafforzamento di queste autorità consisterebbe nel riconoscimento dell'autonomia politica di queste popolazioni.Non è solamente a partire dai rapporti reciproci, comunque elemento preponderante nel quale si cristallizza il senso di autorità, bensì esistono altri elementi, come ad esempio la parentela, la lingua, l'identità (in altri termini, la cultura che da coesione al gruppo), secondo cui chi esercita ed amministra il diritto indigeno s'identifica pienamente con chi lo accetta e asseconda. E chi lo esercita costituisce il mezzo che mette in relazione l'etnia con 1'“esterno".

D'altra parte, è indubbio che l'intensificazione della divisione del lavoro ha determinato che il senso di potere e autorità si "moltiplichi" in forme non propriamente tradizionali, nelle quali compaiono "domini personali" (cacicazgos nella versione originale, n.d.t.), proprietari terrieri o semplicemente forme ricreate dal potere statale, che non corrispondono alla traiettoria culturale indigena.

Però, questa dissomiglianza di poteri ci porta necessariamente a profilare che le concezioni secondo le quali agiscono il potere egemone 
e l'autorità di tipo tradizionale sono contenute in sistemi di valore diversi; così, ad esempio, mentre diversi popoli indigeni cercano di conciliare l'interesse sociale, lo Stato nazionale procura giustizia, attraverso organi specializzati, a chi meglio dimostra le sue ragioni, dandosi il caso assurdo di non riconoscere la "verità reale", bensì la "verità giuridica" (come ad esempio quelle che si originarono nei titoli coloniali che legittimarono la depredazione agraria di milioni di indigeni in tutto il mondo).

Tuttavia, ci sarà da rivalutare il senso coattivo che applica l'autorità indigena, in quanto nel suo agire osserviamo contraddizioni interetniche che in certe occasioni danno un senso di forza per chi applica il diritto indigeno.

In altri casi, le strutture politiche da cui dipende il diritto indigeno possono variare non solo da una regione all'altra ma incluso all'interno di una stessa etnia possono sussistere micro-poteri che a loro volta creano diversi diritti indigeni, aspetti questi che posteriormente potranno essere compiutamente spiegati dall'etnografia (che si appoggerà sullo studio di caso). Ciononostante, il nostro fondamento teorico è quello di rafforzare il riconoscimento di un senso di autorità indigena che non necessariamente sussiste per se -anche se in determinate occasioni si alterna con quella dello Stato- avendo come quadro di legittimazione i rapporti di reciprocità interetnica.

\subsubsection{Linguaggio e Diritto Indigeno}

Nella pratica giuridica indigena, che muove dal principio di oralità, i meccanismi del linguaggio-discorso ${ }^{9}$ acquisiscono uno speciale signifi-

\footnotetext{
9 Come concetto generico di discorso raccolgo la definizione di Charadeau che segnala come: "Il discorso non deve essere assimilato alla manifestazione verbale del linguaggio che ancora si domina nell'insieme delle manifestazioni del linguaggio; corrisponde a un determinato codice semiologico (verbale), vale a dire un codice gestuale (linguaggio del gesto) o un codice iconico (linguaggio dell'immagine). Il linguaggio è al di qua (o al di là) dei codici di manifestazione del linguaggio nel senso in cui è il luogo della messa in scena del significato, il quale può impiegare, per i suoi fini, uno o più codici semiologici”. Riconosciamo che il linguaggio, inteso come discorso, gioca un ruolo primordiale, non solo nell'ambito del diritto indigeno bensì come un nucleo organizzatore dell'identità etnica che dà vita alla cosmovisione dei popoli indigeni. (CHARADEAU, 1980, p. 23)
} 
cato. L'uso di questa categoria, più che adeguarsi a partire da contenuti di tipo assiomatico, cerca di interagire nell' analisi del discorso individuando la logica che sottende al "modello" di amministrazione legale delle popolazioni citate e la loro rispettiva operatività.

Fino ad ora gli studi sulla materia sono precari. Per non cadere in ipotesi semplicistiche daremo una breve descrizione di alcuni aspetti che analizzano il fenomeno.

Rainer Enrique Hamel (p. 20) avverte:

La ricerca socio-linguistica recente sui gruppi indigeni messicani rivela il ruolo specifico che gioca la lingua indigena e, soprattutto, la sua strutturazione discorsiva, in una serie di eventi e attività chiave per l'orientamento socioculturale delle comunità autoctone: processi di lavoro collettivo, lavori, assemblee, conciliazioni, riti religiosi, l'esercizio dell'autorità e del potere in diversi contesti ecc.. In tutte queste istanze appaiono elementi della tradizione giuridica propria delle etnie. Forse però in pochi eventi la stretta relazione tra il diritto consuetudinario e la sua struttura discorsiva si rivela con maggior chiarezza che nelle conciliazioni.

Negli studi di Collier, che s'inquadrano nella risoluzione di conflitti (Zinacantecos México), si è proposto di riprendere il concetto di "Barkwu", intendendo il linguaggio (giuridico) come una legge per condurre e risolvere conflitti. Aspetto complesso, se pretendiamo codici (giuridici-semiologici) molteplici e diversificati, che, essendo l'applicazione di questa normatività basicamente orale, ci condurrebbe a riflettere su quali sono i limiti di suddetta "legge". Il lavoro del ricercatore socio-linguistico dovrebbe piuttosto attenersi ai fenomeni che mettono in relazione la "razionalità indigena" (la loro cultura) con l'organo che, specialmente attraverso il linguaggio giuridico indigeno, applica e rende efficace il discorso stesso. Come sono, ad esempio, in America Latina, le assemblee, i consigli degli anziani, i tatamandones, i principali, i "maggiordomati”, i governatori tradizionali, i consigli supremi eccetera. 
D'altra parte crediamo che l'efficacia e il compimento della normativa indigena hanno un senso di concretezza che non è possibile separare dai codici linguistici conformi alla realtà indigena.

La rottura con i codici linguistici socialmente accettati dalle comunità può orientare l'amministrazione della giustizia indigena verso altre sfere.

Per Santiago Nino (1980, p. 246), la semplice formulazione di norme utilizzando il linguaggio fa sì che si vedano limitate dal problema "di essere applicate con vaghezza, ambiguità e imprecisione, nella misura in cui il senso delle parole non è univoco".

Tuttavia, è importante valutare in che misura si realizza la norma, d'accordo alla forza della tradizione indigena. È per questo limite che, occasionalmente, $\mathrm{i}$ "contendenti" preferiscono ricorrere al diritto positivo come forma abituale di risoluzione.

Cercando di progredire nell'adeguamento del conflitto linguistico soggiacente al diritto indigeno, Rainer Hamel (p. 21) ha proposto il riconoscimento di tre livelli di organizzazione nell'analisi di questo diritto:

1. schemi culturali (sistemi simbolici): concezioni e definizioni di diritti e delitti, procedimenti per le controversie, organizzazione di procedimenti risolutivi, rapporti sociali di rispetto ecc.;

2. strutture e strategie discorsive: linguaggi specialistici vs. quotidiani, strategie verbali, tecniche argomentative e narrative, modelli di interazione verbale eecc.;

3. strutture e forme linguistiche: selezione ed uso delle lingue, cambio di codici, prestiti linguistici, variazioni interne alle lingue e così via.

\subsubsection{Parentela e Diritto Indigeno}

La quinta ed ultima categoria significativa che si collega allo studio del diritto consuetudinario dei popoli indigeni è il sistema di parentela.

A tal effetto ci rifacciamo alla concezione che propone Rodolfo Stavenhagen (1988, p. 100), il quale sostiene: 
I sistemi di parentela, e ne esistono vari fondamentali tra i popoli indigeni dell'America latina, determinano i rapporti tra famiglie, che sono i nuclei basici delle comunità indigene. Hanno anche la funzione di normare i processi di eredità e trasmissione della ricchezza. Occasionalmente, il funzionamento di questi sistemi di parentela entra in contraddizione con il sistema giuridico nazionale. Per esempio, molte società sono poligamiche e le diverse mogli di un capofamiglia svolgono ruoli specifici nella struttura familiare. Ciononostante, la legislazione civile degli stati non riconosce la poligamia e questo può creare seri problemi per la stabilità della famiglia, la posizione dei figli, la trasmissione dei beni in eredità e la posizione, o status, dell'uomo all'interno della comunità.

I rapporti di parentela costituiscono l'asse dei rapporti di produzione, in modo tale che gli usi quotidiani delle famiglie indigene rappresentano l'elemento primigenio di simbolizzazione e vita rituale indigena.

La finalità della famiglia "nucleare" o "estesa", e conseguentemente della famiglia "allargata" (barrio nella versione originale, un concetto che possiamo assimilare appunto alla definizione di f. allargata, con una forte connotazione patriarcale, n.d.t.), direbbero alcuni antropologi del lignaggio, è quella della sussistenza e riproduzione.

A questo scopo stabiliscono una normativa -giuridica- che definisce "equilibri" interni al nucleo familiare. Per soddisfare le necessità materiali è necessario delimitare una struttura gerarchica tra chi organizza/guida e quelli che sono invece guidati.

In questo modo sorgono una serie di diritti e doveri che, senza trovarsi consegnati ad alcun testo, conservano la vita e, conseguentemente, $i$ rapporti sociali della famiglia.

Il principio che guida quest'usanza familiare si esprime in rapporti di reciprocità nei quali ognuno dei membri provvede ad una determinata attività all'interno del nucleo, dandogli coesione e presenza. Gli indigeni sostengono come fondamentali i valori del lavoro che si svolge all'interno del nucleo familiare e della solidarietà tra i suoi membri. 
L'inosservanza di alcune delle attività che corrispondono a ciascuno degli indigeni può portare a stabilire controversie di fronte alle autorità tradizionali o, incluso, di fronte all'ente rappresentativo del governo.

L'organizzazione della struttura familiare si basa sul rispetto agli anziani o ai genitori, che sono quelli che determinano l'azione del nucleo familiare.

\section{Conclusioni}

È possibile, dalla Teoria Generale del Diritto e dall'Antropologia, stabilire lo studio sistematizzato dell'antropologia giuridica, disciplina che si occupa dello studio dell'insieme dei sistemi di diritto indigeno che storicamente hanno regolato i rapporti sociali di questi popoli.

Nel suo senso più generale, questa nuova disciplina -1'antropologia giuridica- si riferisce al profilo dell'essere umano di fronte alla norma giuridica, partendo dal criterio di diversità culturale storicamente sviluppato dai popoli, individuando in questo modo l'esistenza di forme legali ed extra-legali che li regolano.

In particolare, i concetti generali dell'antropologia giuridica che danno fondamento al diritto indigeno derivano dalla cultura di ogni popolo, dove spiccano la parentela, la cosmovisione, il linguaggio e i rapporti di reciprocità che si basano nella terra, non solo come semplice mezzo di produzione ma come un vincolo necessario per la conservazione globale dell'etnia.

Il diritto indigeno, sebbene molteplice, complesso e storico, esiste come un insieme di sistemi di regolazione giuridica che si differenziano dai sistemi di diritto egemoni. Le strutture ed il processo in cui si basano $i$ sistemi di diritto indigeno, lo sostentano come una normazione ad hoc per la razionalità indigena, in quanto è efficace nella sua applicazione all'adeguarsi ai modelli culturali di ogni etnia. Le nuove definizioni democratiche sulle quali si baserà la post-modernità dovranno sentire la necessità del riconoscimento dei diritti indigeni, come dei loro organi esecutori. 


\section{Riferimenti}

ALCIDES REISSNER, Raúl. El indio en los diccionarios. Messico: INI, 1980.

CARBONIER, Jean. Sociologie juridique. Parigi: PUF, 1978.

CHARADEAU, Patrick.Una teoría de los sujetos del lenguaje. In:

Discurso. Centre d'Analyse du Discours. Università di Parigi XIII. Parigi: UNAM, 1980.

CLASTRES, Pierre.Anty-Mythe.Rivista Civilización. p. 48, bis, set. 1984.

DAI CHA FOU. Las nacionalidades en China: demografía y política. In: Etnias de Oriente y Occidente. Messico: CONACULTA, 1991. p. 7992.

DAVID, Renè; JAUFFRET-SPINOSI, Camille. Les grands systèmes de droit contemporains. Parigi: Dalloz, 1988.

GONZÁLEZ GALVÁN, Jorge. Derecho indígena. Messico: Harla/ UNAM, 1996.

FABIG, Heikee; VEREYEN, Luc. In heemsen. In de Uvvrlijn van de Ontwikkeling. Wereld Morgen. MAANBLAD

VOOR INTERNATIONALE VERSTENDHOUDING EN

ONTWIKKELINGSSAMEN WORKING, Bruxelles, (29), n. 5, 1993.

HUMAN RIGHTS.INDIGENOUS PEOPLES AND SLAVERY IN THE UNITED NATIONS. A special report of two working groups of the Human Rights, Sub-Commission,Ottawa, ago. 1991.

KRAWIETZ, Werner.Zum paradigmenwchsel methodenstreit. In: KRAWIETZ; OPALEK; PECZENIK. Argumentation und hermeneutik in der jurisprudenz. Berlino, 1979.

MARX, Carl. Elementos fundamentales para la crítica de la economía política (Grundrisse) 1857-1858. 13ª ristampa. Messico:Siglo XXI, 1984. 
NINO, Carlos S. Introducción al análisis del derecho. Buenos Aires: Astrea, 1980.

HAMEL, Rainer E. Costumbre jurídica y lenguaje. In: América indígena, n. 25.

RIZO, Mario. Consideraciones acerca del ordenamiento consuetudinario en las comunidades indígenas de las Regiones Autónomas del Atlántico Norte. In: IV LABORATORIO DI ANTROPOLOGIA CENTROAMERICANA. Managua, Nicaragua, 16-18, apr. 1991. STAVENHAGEN, Rodolfo. Derecho indígena y derechos humanos. Messico: Colegio de México, 1988.

Carlos Humberto Durand Alcántara Dottore di Antropologia Giuridica - UNAM/Mexico. Postdottore negli Studi di Sociologia Rurale e Contadine Universidad de Córdoba/España. Coordinatore del Proyecto de Maestría en Derecho de la Universidad Autónoma Metropolitana-Azcapotzalco. Ricercatores Nazionale del Messico - Livello II - CONACYT - SNI. Coordenador da Red Internacional en Pobreza y Pueblos Indios. Capo del Área de Investigación en Derechos Humanos y Alternatividad Jurídico Social.E-mail: carloshdurand@ yahoo.com.mx.

Endereço profissional: Calle Niños Héroes, n. 205. Col Santa Maria Tepepan Xocimiilco. México - Distrito Federal - Código POSTAL 16020. 